周邊が固定され、直交彈性梁に乘る矩形板

の解法及實驗に就て

正員工學士政 井忠二

Theory and Experiment on the Rectangular Plate clamped at

the Periphery and Supported by Many Elastic Beams

under Uniformly Distributed Load

By Chûji FuJr, Kôgakusi, Member

摘 要

著者は先に本會の會誌 ${ }^{(2)}$ に、周邊が目由に支へられたる矩形板が彈性梁に乘る場合の近似的解法に就て發 表し、又其後此種の一二の問題に就て講演した事が有る。本論文は之等の解涯を今少し延長して多少一般的 に取抜つたもので、其計算及實驗例として 周邊が固定された場合をとつた。本計算は可成複雜にはなるが、 其結果は實驗結果と良く一致する事を確めた。

\section{1. 基本微分方程式}

矩形板が彈性梁に依つて支へられて 居る場合、之等兩 者の接觸面には或力が呼起される。此力は彈性梁に就て 云へば夫を變形させるに必要な外力で有り、矩形板に就 て云へば其支持力で有り、文同時に夫に働く外力とも看 做し得る。偖此彈性梁に及ぼす力と矩形板に及ぼす力と は云ふ迄もなく方向反對で等大で有るから、彈性梁を取 去り其代りに彈性梁に作用子る力に等しい外力が矩形板 に作用するものと考へれば、我々は此種の問題を複雜な 荷重を受ける矩形板の問題に誘導する事が出來る。

今矩形板の邊の長さを夫及 $a$ 及 $b$ とし、直交軸 $x$ 及 $y$ を第 1 圖の如くとり、彈性梁は圖中默線で示せる如 く矩形板の中心線に對して左右對秲に配置されて居て、 之等の $x$ 及 $y$ 軸からの距離は夫々圖中に記入せる如き

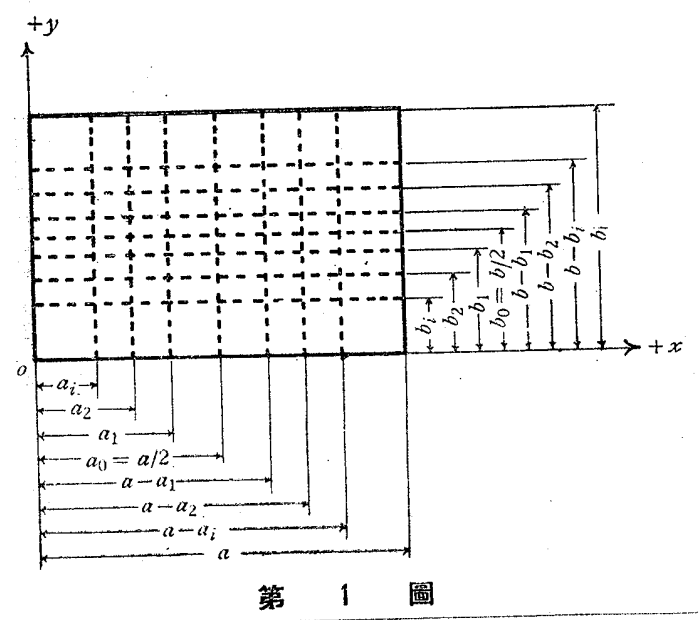

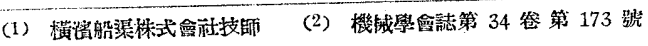

わので有るとする。今 $x=a_{i}$ 及 $y=b_{i}$ に於ける彈性 梁の任意の箅所に作用する外力を夫々 $p_{a_{i}}$ 及 $p_{b_{i}}$ とせば 之等は次の樣に書き表せる。

$$
p_{a_{i}}=E_{a_{i}} I_{a_{i}} \cdot \frac{d^{4} w_{x=a_{i}}}{d y^{4}}, p_{b i}=E_{b i} I_{b i} \cdot \frac{d^{4} w_{y=b i}}{d x^{4}} \cdots
$$

但上式中 $E_{a_{i}}, E_{b_{i}}$ 及 $I_{a_{i}}, I_{b i}$ は夫及 $x=a_{i}$ 及 $y=b_{i}$ に於ける彈性梁の彈性係數及慣性能率、 $w$ は矩形 板の摬み、 $w_{x=a_{i}}$ 及 $w_{y=b_{i}}$ は $w$ の式に $x=a_{i}$ 及 $y=b_{i}$ を代入したもので、夫々 $x=a_{i}$ 及 $y=b_{i}$ に於


相當する式を導く事吕出來る。從つて前述せる如く之等 の力が外力として矩形板に作用寸るので有るから、唯今 の場合の微分方程式は一般に次の如く書ける。

$$
\begin{aligned}
& \frac{\partial^{4} w}{\partial x^{4}}+2 \frac{\partial^{4} w}{\partial x^{2} \partial y^{2}}+\frac{\partial^{4} w}{\partial y^{4}} \\
& =\frac{1}{N}\left\{p-E_{a:} I_{a 0} \cdot \frac{d^{4} w_{x=a_{0}}}{d y^{4}}-E_{b j} I_{b j} \frac{d^{4} w_{y=b_{J}}}{d x^{4}}\right. \\
& \left.-2 \cdot \sum_{1,9_{\ldots} \ldots}^{i} E_{a_{i}} I_{a_{i}} \cdot \frac{d^{4} w_{x=a_{i}}}{d y^{4}}-2 \cdot \sum_{1,2 \ldots}^{i} E_{b_{i}} I_{b_{i}} \frac{d^{4} w_{y=b i}}{d x^{4}}\right\} \\
& \text { 但 } N=\frac{E h^{3}}{12\left(1-\nu^{2}\right)}
\end{aligned}
$$

本式中 $E, h$ 灭 $v$ は夫及矩形板の彈性倸數、板の厚み 攻ポアッッン比で准る。郎上:式の解式を求め其中に含ま れる未知係數が周邊の條件を滿足する樣に決定され」 ば、此處に問題は解決するので有る。然し乍ら此種の問 題に斯る解法を用ひる事は非常に因難で有るから、著者 は寧ろ周邊の條件を滿足する如き或函數を撰び、此式が （2）を滿足する樣に未知係數を決定する方法を提唱する。 [第 35 管 185 號 
2. 周邊が固是或は自由に、攵は之等兩者中間の任意の狀態に支持され、幾多の 道交彈性梁で支へられて居る矩形板が一樣な散布衔重を受ける場合

壯稳の條件を考慮して撓みの式を次の樣に假定して見る

$$
\begin{aligned}
& w=\frac{p_{0} a^{4}}{N} \sum_{m}^{\infty} \sum_{n}^{\infty} \frac{A_{m n}}{\left(m^{2}+\lambda^{2} n^{2}\right)^{2}}\left\{\frac{1}{m \pi} \sin \frac{m \pi x}{a}+\alpha\left(\frac{x^{2}}{a^{2}}-\frac{x}{a}\right)\right\}\left\{\frac{1}{n \pi} \sin \frac{n \pi y}{b}+\beta\left(\frac{y^{2}}{b^{2}}-\frac{y}{b}\right)\right\} \\
& \text { 但 } m, n=1,3,5, \cdots \cdots \\
& \lambda=a / b
\end{aligned}
$$

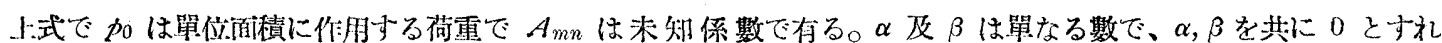
ば周邊が自由に支いられた場合となり、之等を共に 1 とすれば周邊が完全に固䇥された場合となる、從つて $\alpha$ 及 $\beta$ を夫ト 0 と 1 の間の任意の值を與へる事に依つて、任意の周邊の條件を滿足さ寸事が出來る。偖此處に一寸瞇づ 置きたい事は、若し彈性梁が矩形板に比して非掌に丈夫に出來て居て、梁そ梁との間隔が板の厚みに比して大なる場 合には、唕性平面は一つの滑らかな曲面とは成らずに、幾多の波を持つ曲面に成る事が想像出來るので有るが、唯今 は其影響は極く僅少で一つの滑らかな曲面に成るものと假定する點で有る。

偖以上の如き假定が成立すれば（4）ほ必ずしも不當な假定では無から5。今（4）を(2)に代入して計算すれば 次の樣になる。

$$
\begin{aligned}
& \frac{p_{0} \pi^{3}}{N} \sum_{m}^{\infty} \sum_{n}^{\infty} A_{m n} \frac{m^{3}}{\left(m^{2}+\lambda^{2} n^{2}\right)^{2}} \cdot \sin \frac{m \pi x}{a} \cdot\left[\frac{1}{n \pi} \sin \frac{n \pi y}{b}+\beta\left(\frac{y^{2}}{b^{2}}-\frac{y}{b}\right)\right] \\
& +\frac{2 p_{0} \lambda^{2}}{N} \sum_{m}^{\infty} \sum_{n}^{\infty} A_{m n} \frac{1}{\left(m^{2}+\lambda^{2} n^{2}\right)^{2}} \cdot\left[-m \pi \cdot \sin \frac{m \pi x}{a}+2 \alpha\right]\left[-n \pi \cdot \sin \frac{n \pi y}{b}+2 \beta\right] \\
& +\frac{p_{0} \lambda^{4} \pi^{3}}{N} \sum_{m}^{\infty} \sum_{n}^{\infty} A_{m n} \frac{n^{3}}{\left(m^{2}+\lambda^{2} n^{2}\right)^{2}}\left[\frac{1}{m \pi} \sin \frac{m \pi x}{a}+\alpha\left(\frac{x^{2}}{a^{2}}-\frac{x}{a}\right)\right] \cdot \sin \frac{n \pi y}{b} \\
& =\frac{p_{0}}{N}-\frac{E_{a_{j}} I_{a j}}{N} \cdot \frac{p^{0} a^{4}}{N} \sum_{m}^{\infty} \sum_{n}^{\infty} \frac{A_{m n}}{\left(m^{2}+\lambda^{2} n^{2}\right)^{2}}\left[\frac{\sin \frac{m \pi}{2}}{m \pi}-\frac{\alpha}{4}\right] \cdot \frac{1}{n \pi}\left(\frac{n \pi}{b}\right)^{4} \cdot \sin \frac{n \pi y}{b} \\
& -\frac{E_{b 3} I_{b j}}{N} \cdot \frac{p_{0} a^{4}}{N} \sum_{m}^{\infty} \sum_{n}^{\infty} \frac{A_{m n}}{\left(m^{2}+\lambda^{2} n^{2}\right)^{2}} \cdot \frac{1}{m \pi}\left(\frac{m \pi}{a}\right)^{4} \sin \frac{m \pi x}{a} \cdot\left[\frac{1}{n \pi} \sin \frac{n \pi}{2}-\frac{\beta}{4}\right] \\
& -2 \cdot \sum_{3,2 \ldots}^{i} \frac{E_{a i} I_{a i}}{N} \cdot \frac{p_{0} a^{4}}{N} \sum_{m}^{\infty} \sum_{n}^{\infty} \frac{A_{m n}}{\left(m^{2}+\lambda^{2} n^{2}\right)^{2}} \cdot\left[-\frac{\sin \frac{m \pi a_{i}}{a}}{m \pi}+\alpha\left(\frac{a_{i}^{2}}{a^{2}}-\frac{a_{i}}{a}\right)\right] \cdot \frac{1}{n \pi}\left(\frac{n \pi}{b}\right)^{4} \sin \frac{n \pi y}{b} \\
& -2 \cdot \sum_{1,2 \ldots}^{i} \frac{E_{b_{i}} I_{b i}}{N} \cdot \frac{p_{0} a^{4}}{N} \sum_{m}^{\infty} \sum_{n}^{\infty} \frac{A_{m n}}{\left(m^{2}+\lambda^{2} n^{2}\right)^{2}} \cdot \frac{1}{m \pi} \cdot\left(\frac{m \pi}{a}\right)^{4} \sin \frac{m \pi x}{a} \cdot\left[\frac{\sin \frac{n \pi b_{i}}{b}}{n \pi}+\beta\left(\frac{b_{i}{ }^{2}}{b^{2}}-\frac{b_{i}}{b}\right)\right] \ldots
\end{aligned}
$$

更に上式を適當にフリェーの級數に屡開して、多少の書換へを施せば次の樣になる

$$
\begin{aligned}
& \frac{p_{0} \pi^{2}}{N} \sum_{m}^{\infty} \sum_{n}^{\infty} A_{m n} \cdot \frac{1}{m n} \sin \frac{m \pi x}{a} \sin \frac{n \pi y}{b} \\
& -\frac{8 p_{0} \beta}{N} \sum_{m}^{\infty} \sum_{n}^{\infty} \sum_{q}^{\infty} A_{m q}\left[\frac{m^{3}}{n^{3}\left(m^{2}+\lambda^{2} q^{2}\right)^{2}}+\frac{2 \lambda^{2} m}{n\left(m^{2}+\lambda^{2} q^{2}\right)^{2}}\right] \cdot \sin \frac{m \pi x}{a} \cdot \frac{n \pi y}{b} \\
& -\frac{8 p_{0} \lambda^{2} \alpha}{N} \sum_{m}^{\infty} \sum_{n}^{\infty} \sum_{p}^{\infty} A_{p n}\left[\frac{\lambda^{2} n^{3}}{m^{3}\left(p^{2}+\lambda^{3} n^{2}\right)^{2}}+\frac{2 n}{m\left(p^{2}+\lambda^{2} n^{2}\right)^{2}}\right] \cdot \sin \frac{m \pi x}{a} \cdot \sin \frac{n \pi y}{b} \\
& -\frac{128 p_{0} \lambda^{2} \alpha \beta}{N \pi^{2}} \sum_{m}^{\infty} \sum_{n}^{\infty} \sum_{p}^{\infty} \sum_{q}^{\infty} A_{p q} \frac{1}{m n\left(p^{2}+\lambda^{2} q^{2}\right)^{2}} \cdot \sin \frac{m n x}{a} \cdot \sin \frac{n \pi y}{b}
\end{aligned}
$$

昭和 7 年 9 月] 


$$
\begin{aligned}
& =\frac{16 p_{0}}{N \pi^{2}} \sum_{m}^{\infty} \sum_{n}^{\infty} \frac{1}{m n} \sin \frac{m \pi x}{a} \cdot \sin \frac{n \pi y}{b} \\
& -\frac{2 p_{0} \lambda^{3} \pi^{3}}{N} \sum_{m}^{\infty} \sum_{n}^{\infty} \sum_{p}^{\infty} A_{m} \frac{n^{3}}{\left(p^{2}+\lambda^{2} n^{2}\right)^{2}}\left\{k_{a 0}\left(\frac{\sin \frac{p \pi}{2}}{p \pi}-\frac{\alpha}{4}\right) \sin \frac{m \pi}{4}\right. \\
& \left.+\sum_{1, \cdots \ldots}^{i} k_{a i}\left[\frac{\sin \frac{p_{\pi} a_{i}}{a}}{p_{\pi}}+\alpha\left(\frac{a_{i}{ }^{2}}{a^{2}}-\frac{a_{i}}{a}\right) \sin \frac{m \pi a_{i}}{a}\right]\right\} \sin \frac{m \pi x}{a} \sin \frac{n \pi y}{b} \\
& -\frac{2 p_{0} \pi^{3}}{N} \sum_{m}^{\infty} \sum_{n}^{\infty} \sum_{q}^{\infty} A_{m q} \frac{m^{3}}{\left(m^{2}+\lambda^{2} n^{2}\right)^{2}}\left\{k_{b 0}\left(\frac{\sin \frac{q \pi}{2}}{q \pi}-\frac{\beta}{4}\right) \sin \frac{n \pi}{2}\right. \\
& \left.+\sum_{1,2 \ldots}^{i} k_{b_{i}}\left[\frac{\sin \frac{q \pi b_{i}}{b}}{q \pi}+\beta\left(\frac{b_{i}^{2}}{b^{2}}-\frac{b_{i}}{b}\right) \sin \frac{n \pi b_{i}}{b}\right]\right\} \sin \frac{m \pi x}{a} \cdot \sin \frac{n \pi y}{b} \\
& \text { 但 } \quad p, q=1,3,5, \cdots \\
& k_{a 3}=\frac{E_{a}, I_{a_{0}}}{b N}, \quad k_{a_{i}}=\frac{E_{a_{i}} I_{a_{i}}}{b N} \\
& k_{b_{3}}=\frac{E_{b_{0}} I_{b_{0}}}{b N}, \quad k_{b_{i}}=\frac{E_{b_{i}} I_{b_{i}}}{b N},
\end{aligned}
$$

從つて上式が成立する䉆めには未知係數 $A_{m n}$ が次の關係式を滿足せねばならぬ

$$
\begin{aligned}
& A_{m n} \cdot \frac{\pi^{2}}{m n}-8 \beta \sum_{p}^{\infty} A_{m q}\left[\frac{m^{3}}{n^{3}\left(m^{2}+\lambda^{2} q^{2}\right)^{2}}+\frac{2 m}{n\left(m^{2}+\lambda^{2} q^{2}\right)^{2}}\right] \\
& -8 \lambda \alpha \sum_{p}^{\infty} A_{p n}\left[\frac{\lambda^{2} n^{3}}{m^{3}\left(p^{2}+\lambda^{2} n^{2}\right)^{2}}+\frac{2 n}{m\left(p^{2}+\lambda^{2} n^{2}\right)^{2}}\right]+\frac{128 \lambda^{2} \alpha \beta}{\pi^{2}} \sum_{p}^{\infty} \sum_{q}^{\infty} A_{p q} \frac{1}{m n\left(p^{2}+\lambda^{2} q^{2}\right)^{2}} \\
& =\frac{16}{\pi^{2} m n}-2 \lambda^{3} \pi^{3} \sum_{p}^{\infty} A_{p n} \frac{n^{3}}{\left(p^{2}+\lambda^{2} n^{2}\right)^{2}}\left\{k_{a j}\left(\frac{\sin \frac{p \pi}{2}}{p \pi}-\frac{\alpha}{4}\right) \sin \frac{m \pi}{a}\right. \\
& \left.+\sum_{1,2 \ldots}^{i} k_{a_{i}}\left[\frac{\sin \frac{p \pi a_{i}}{a}}{p \pi}+\alpha\left(\frac{a_{i}^{2}}{a^{2}}-\frac{a_{i}}{a}\right)\right] \sin \frac{m \pi \alpha_{i}}{a}\right\} \\
& -2 \pi^{3} \sum_{q}^{\infty} A_{m q} \frac{m^{3}}{\left(m^{2}+\lambda^{2} q^{2}\right)^{2}}\left\{k_{l 0}\left(\frac{\sin \frac{q \pi}{2}}{q \pi}-\frac{\beta}{4}\right) \sin \frac{n \pi}{2}\right. \\
& \left.+\sum_{1, \cdots \ldots}^{i} k_{b_{i}}\left[\frac{\sin \frac{q \pi b_{i}}{b}}{q \pi}+\beta\left(\frac{b_{i}{ }^{2}}{b}-\frac{b_{i}}{b}\right)\right] \sin \frac{n \pi b_{i}}{b}\right\}
\end{aligned}
$$

郎之が未知係數 $A_{m n}$ 足決定する條件式で有る。偖上式より $A_{m n}$ が求まれば、撓みの式 (4) が完全に決定された 事になり、從つて又彎曲偶力、垂直應力及其他 (3) のものも求める事が出來る。之等の內最も大切な役割を成するのは 勿椧絽曲偶力(又は垂直應力)で有るから、唯今は他を省略して、之を求むる式のみを次に導い、置く。 


$$
\begin{aligned}
& M_{x}=-p_{0} a^{2} \sum_{m}^{\infty} \sum_{n}^{\infty} \frac{A_{m n}}{\left(m^{2}+\lambda^{2} n^{2}\right)^{2}}\left[f(m, x) \cdot \varphi(n, y)+\nu \lambda^{2} \cdot \varphi(m, x) \cdot f(n, y)\right] \\
& \left.M_{y}=-p_{0} a^{2} \sum_{m}^{\infty} \sum_{n}^{\infty} \frac{A_{m n}}{\left(m^{2}+\lambda^{2} n^{2}\right)^{2}}\left[\lambda^{2} \cdot \varphi(m, x) \cdot R(n, y)+\nu \cdot f(m, x) \cdot \varphi(n, y)\right]\right] \\
& \text { 但 } f(m, x)=2 x-m \pi \cdot \sin \frac{m \pi x}{a} \\
& f(n, y)=2 \beta-n \pi \cdot \sin \frac{n \pi y}{b} \\
& \varphi(m, x)=\frac{1}{m \pi} \cdot \sin \frac{m \pi x}{a}+\alpha\left(\frac{x^{2}}{a^{2}}-\frac{x}{a}\right) \\
& \varphi(n, y)=\frac{1}{n \pi} \cdot \sin \frac{n \pi y}{b}+\beta\left(\frac{y^{2}}{b^{2}}-\frac{y}{b}\right) \\
& M_{a j}=-p_{0} a^{2} b \lambda k_{a j} \sum_{m}^{\infty} \sum_{n}^{\infty} \frac{A_{m n}}{\left(m^{2}+\lambda^{2} n^{2}\right)^{2}}\left[\frac{\sin \frac{m \pi}{2}}{m \pi}-\frac{\alpha}{4}\right]\left[2 \beta-n \pi \cdot \sin \frac{n \pi y}{b}\right] \\
& M a_{1}=-p_{0} a^{2} b \lambda k a_{1} \sum_{m}^{\infty} \sum_{n}^{\infty} \frac{A_{m n}}{\left(m^{2}+\lambda^{2} n^{2}\right)^{2}}\left[\frac{\sin \frac{m \pi a_{i}}{a}}{m \pi}+\alpha\left(\frac{a_{1}^{2}}{a^{2}}-\frac{a_{1}}{a}\right)\right]\left[2 \beta-n \pi \cdot \sin \frac{n \pi y}{b}\right] \text { \} } \\
& \left.M_{a_{i}}=-p_{0} a^{2} b \lambda k_{a_{1}} \sum_{m}^{\infty} \sum_{n}^{\infty} \frac{A_{m n}}{\left(m^{2}+\lambda^{2} n^{2}\right)^{2}}\left[\frac{\sin \frac{m \pi a_{i}}{a}}{m \pi}+\alpha\left(\frac{a_{i}^{2}}{a^{2}}-\frac{a_{i}}{a}\right)\right]\left[2 \beta-n \pi \cdot \sin \frac{n \pi y}{b}\right]\right) \\
& \left.M_{b_{0}}=-p_{0} a^{2} b k_{b}\right) \sum_{m}^{\infty} \sum_{n}^{\infty} \frac{A_{m n}}{\left(m^{2}+\lambda^{2} n^{2}\right)^{2}}\left[2 \alpha-m \pi \cdot \sin \frac{m \pi x}{a}\right]\left[\frac{\sin \frac{n \pi}{2}}{n \pi}-\frac{\beta}{4}\right] \\
& M_{b_{1}}=-p_{0} a^{2} b k_{b_{1}} \sum_{m}^{\infty} \sum_{n}^{\infty} \frac{A_{m n}}{\left(m^{2}+\lambda^{2} n^{2}\right)^{2}}\left[2 \alpha-m \pi \cdot \sin \frac{m \pi x}{a}\right]\left[\frac{\sin \frac{n \pi b_{1}}{b}}{n \pi}+\beta\left(\frac{b_{1}^{2}}{b^{2}}-\frac{b_{1}}{b}\right)\right] \\
& \left.M_{b_{i}}=-p_{0} a^{2} b k_{b_{i}} \sum_{m}^{\infty} \sum_{n}^{\infty} \frac{A_{m n}}{\left(m^{2}+\lambda^{2} n^{2}\right)^{2}}\left[2 \alpha-m \pi \cdot \sin \frac{m \pi x}{a}\right]\left[\frac{\sin \frac{n \pi b_{i}}{b}}{n \pi}+\beta\left(\frac{b_{i}^{2}}{b^{2}}-\frac{b_{i}}{b}\right)\right]\right)
\end{aligned}
$$

此處に $M_{x}, M_{y}$ は矩形板の彎曲偶力で、 $M_{a_{0}}, M_{a_{1}}, \cdots M_{a_{i}}$ 及 $M_{b_{0}}, M_{b_{1}} \cdots M_{b_{i}}$ は夫々 $x=a_{0}, x=a_{1} \cdots x=a_{i}$ 及 $y=b_{0}, y=b_{1}, \cdots y=b_{i}$ に於柱る彈性梁の彎曲偶力で有る。

3. 周邊が完全に固定され、一栐な散布街重を受ける正方形板が、十字形 の彈性梁で支へられて居る場合の計算例



第 2 圆
計算の一例として、 $\lambda=a \mid b=1, k_{a_{0}}=k_{b_{0}}=k, k_{a_{1}}=k_{a_{2}}=\cdots=k_{a i}=0, k_{u_{1}}=$

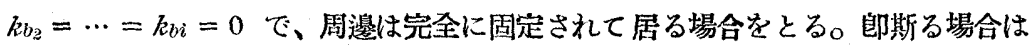
第 2 圆に示技く、板は正方形で、彈性梁は唯 2 本で之等が板の中央で十字に 值交する場合で有る。此際未知係數 $A_{m n}$ を決定する條件式は次の樣になる。

$$
\begin{aligned}
& A_{m n}-\frac{8}{\pi^{2}} \sum_{q}^{\infty} A_{m q} \cdot\left(\frac{m^{4}}{n_{2}}+2 m^{2}\right) \cdot \frac{1}{\left(m^{2}+q^{2}\right)^{2}} \\
& -\frac{8}{\pi^{2}} \sum_{p}^{\infty} A_{p m}\left(\frac{n^{4}}{m^{2}}+2 n^{2}\right) \cdot \frac{1}{\left(p^{2}+n^{2}\right)^{2}}+\frac{128}{\pi^{4}} \sum_{p}^{\infty} \sum_{q}^{\infty} A_{m q} \frac{1}{\left(p^{2}+q^{2}\right)^{2}}
\end{aligned}
$$

昭和 7 年 9 月了 


$$
\begin{aligned}
=\frac{16}{\pi^{4}}-2 \pi k \sum_{q}^{\infty} A_{m q} \frac{m n^{4} n}{\left(m^{2}+q^{2}\right)^{2}}\left(\frac{\sin \frac{q \pi}{2}}{q \pi}-\frac{1}{4}\right) \cdot \sin \frac{n \pi}{2} \\
\\
-2 \pi k \sum_{p}^{\infty} A_{m m} \frac{m n^{4}}{\left(p^{2}+n^{2}\right)^{2}}\left(\frac{\sin \frac{p \pi}{2}}{p \pi}-\frac{1}{4}\right) \cdot \sin \frac{m \pi}{2}
\end{aligned}
$$

偖次節に示す實驗例の場合と同一に $\nu=0.3, k=0.14415$ として、上式より $A_{m n}$ を哔算すれ机次の如くになる。

$$
\begin{array}{r}
A_{11}=+1.5505 \\
A_{13}=A_{31}=+2.0673 \\
A_{33}=+0.5051 \\
A_{15}=A_{51}=-2.3166 \\
A_{35}=A_{53}=-0.6486 \\
A_{55}=-1.2967
\end{array}
$$

此處に唯今の場合の撓み及戀曲偶力の諸式を今一度書い見ると次の樣になる。

$$
\begin{aligned}
& w=\frac{p_{0} a^{4}}{N} \sum_{m}^{\infty} \sum_{n}^{\infty} \frac{A_{m n}}{\left(m^{2}+n^{2}\right)^{2}}\left\{\frac{1}{m \pi} \sin \frac{m \pi x}{a}+\left(\frac{x^{2}}{a^{2}}+\frac{x}{a}\right)\right\}\left\{\frac{1}{n \pi} \sin \frac{n \pi y}{a}+\left(\frac{y^{2}}{a^{2}}-\frac{y}{a}\right)\right\} \\
& M_{x}=-p_{0} a^{2} \sum_{m}^{\infty} \sum_{n}^{\infty} \frac{A_{m n}}{\left(m^{2}+n^{2}\right)^{2}}\left[f_{1}(m, x) \cdot \varphi_{1}(n, y)+\nu \varphi_{1}(m, x) \cdot f_{1}(n, y)\right] \\
& M_{y}=-p_{0} a^{2} \sum_{m}^{\infty} \sum_{n}^{\infty} \frac{A_{m n}}{\left(m^{2}+n^{2}\right)^{2}}\left[\varphi_{1}(m, x) \cdot f_{1}(n, y)+\nu f_{1}(m, x) \cdot \varphi_{1}(n, y)\right] \\
& \text { 但 } f_{1}(m, x)=2-m \pi \sin \frac{m \pi x}{a} \\
& f_{1}(n, y)=2-n \pi \cdot \sin \frac{n \pi y}{a} \\
& \varphi_{1}(m, x)=\frac{1}{m \pi} \sin \frac{m \pi x}{a}+\left(\frac{x^{2}}{a^{2}}-\frac{x}{a}\right) \\
& \varphi_{1}(n, y)=\frac{1}{n \pi} \sin \frac{n \pi y}{a}+\left(\frac{y^{2}}{a^{2}}-\frac{y}{a}\right) \\
& M_{a}=-p_{0} a^{3} k \sum_{m}^{\infty} \sum_{n}^{\infty} \frac{A_{m n}}{\left(m^{2}+n^{2}\right)^{2}}\left[\frac{\sin \frac{m \pi}{2}}{m \pi}-\frac{1}{4}\right]\left[2-n \pi \cdot \sin \frac{n \pi y}{a}\right] \\
& M_{b_{3}}=-p_{0} a^{3} k \sum_{m}^{\infty} \sum_{n}^{\infty} \frac{A_{m n}}{\left(m^{2}+n^{2}\right)^{2}}\left[2-m \pi \cdot \sin \frac{m \pi x}{a}\right]\left[\frac{\sin \frac{n \pi}{2}}{n \pi}-\frac{1}{4}\right] .
\end{aligned}
$$

今（15）の $A_{m n}$ の值を上の諸式に入れて、撓み及彎曲偶力を求めると第 1 表及第 2 表の如くなる。

第 1 表 撓队

\begin{tabular}{c|c|c||c|c|c}
\hline$x / a$ & $y / a$ & $z \cdot\left(\frac{p_{0} a^{4}}{N}\right)^{-1}$ & $x / a$ & $y / a$ & $z \cdot\left(\frac{p_{0} a^{4}}{N}\right)^{-1}$ \\
\hline $1 / 8$ & $4 / 8$ & 0.000232 & $2 / 8$ & $3 / 3$ & 0.000563 \\
$2 / 8$ & $"$ & 0.000620 & $3 / 8$ & $"$ & 0.000813 \\
$3 / 8$ & $"$ & 0.000870 & $1 / 8$ & $2 / 8$ & $0 \cdot 000151$ \\
$4 / 8$ & $\#$ & 0.000978 & $2 / 8$ & $"$ & 0.000451 \\
$1 / 8$ & $3 / 8$ & 0.000213 & $1 / 8$ & $1 / 8$ & 0.0000566 \\
\hline
\end{tabular}

第 2 衰 彎曲嘱力 $M_{x}, M_{y}$ 及 $M_{b_{0}}$

\begin{tabular}{c|c|c|c|c}
\hline$x / a$ & $y / a$ & $M_{x} \cdot\left(p_{0} a^{2}\right)^{-1}$ & $M_{y} \cdot\left(p_{0} a^{2}\right)^{-1}$ & $M_{b_{0} \cdot\left(p_{0} a^{2}\right)^{-1}}$ \\
\hline 0 & $1 / 2$ & -0.03935 & -0.01180 & -0.005672 \\
$1 / 8$ & $"$ & -0.008076 & -0.000785 & -0.002242 \\
$2 / 8$ & $"$ & +0.008826 & +0.008659 & +0.0009866 \\
$3 / 8$ & $"$ & +0.01527 & +0.01389 & +0.001785 \\
$4 / 8$ & $"$ & +0.01626 & +001626 & +0.001803 \\
\hline
\end{tabular}

[第 35 卷第 185 號 


\section{4. 實 驗 例}

以上の計算結果が如何なる 程度に賔驗結果と一致する か確めて見る事にした。，實驗に使用せる正方形板及彈性 梁の材質は共に同一で、 $v=0.3$ と假定し、郘性係數は $E=2.116 \times 10^{6} \mathrm{~kg} / \mathrm{cm}^{2}$ で有つた。板の寸洗は $a=b=$ $201.8 \mathrm{~mm} 、 h=2.817 \mathrm{~mm}$ で、又梁の寸法は第 3 圖に示 して置いた。此際梁の慣性能率は $I=0.005961 \mathrm{~cm}^{4}$ て、 從つて $k$ の值は前篩に記載せる如く $k=0.14415$ とな る。之等の板及梁を第 4 圖及寫貝 $\mathrm{A}$ に示す樣に取付 け、寫覓 $B$ に示寸裝置で一樣な散布荷重を加へて攃み を測定した。撓みの測定裴置は第 5 圖に示す如き裝置 に体り、其精度を $1 / 1000 \mathrm{~mm}$ とした。此實驗で最も大 切で且困難な點は、板の仕上にまたつて出來るだけ曲り



第 3 圖 彈 性 梁
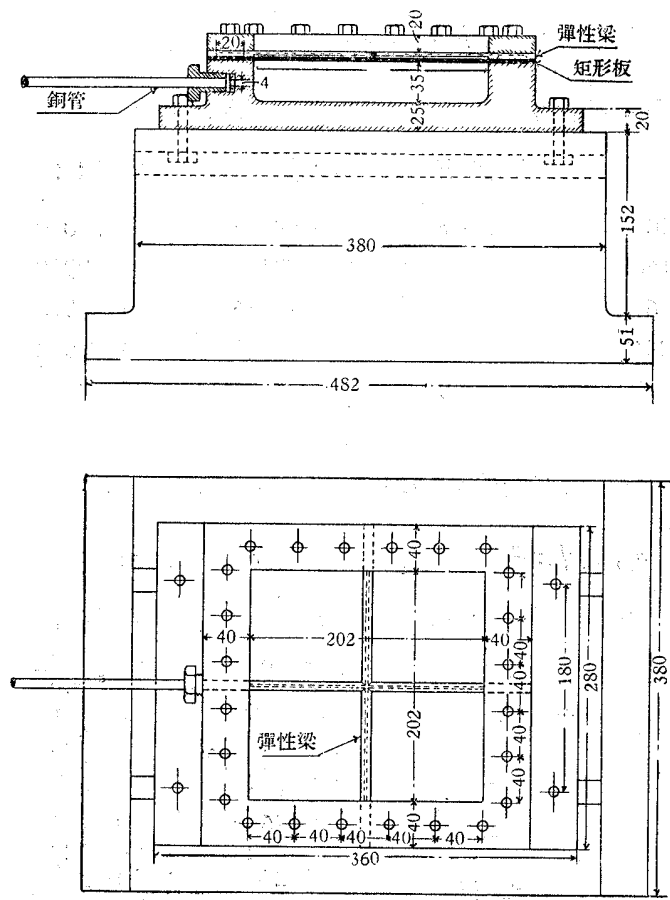

第 4 圖 蕒驗装置
の出ない㨾にして、愿みを一樣にする事、板と梁の接觸 が良い事及其取付が完全固定に最も近い狀態になる事等 て、之等三條件を滿足さ寸爲めに可成苦心し、大牛は長 時間の䅛合により目的に近いるのを得る事が出來た。
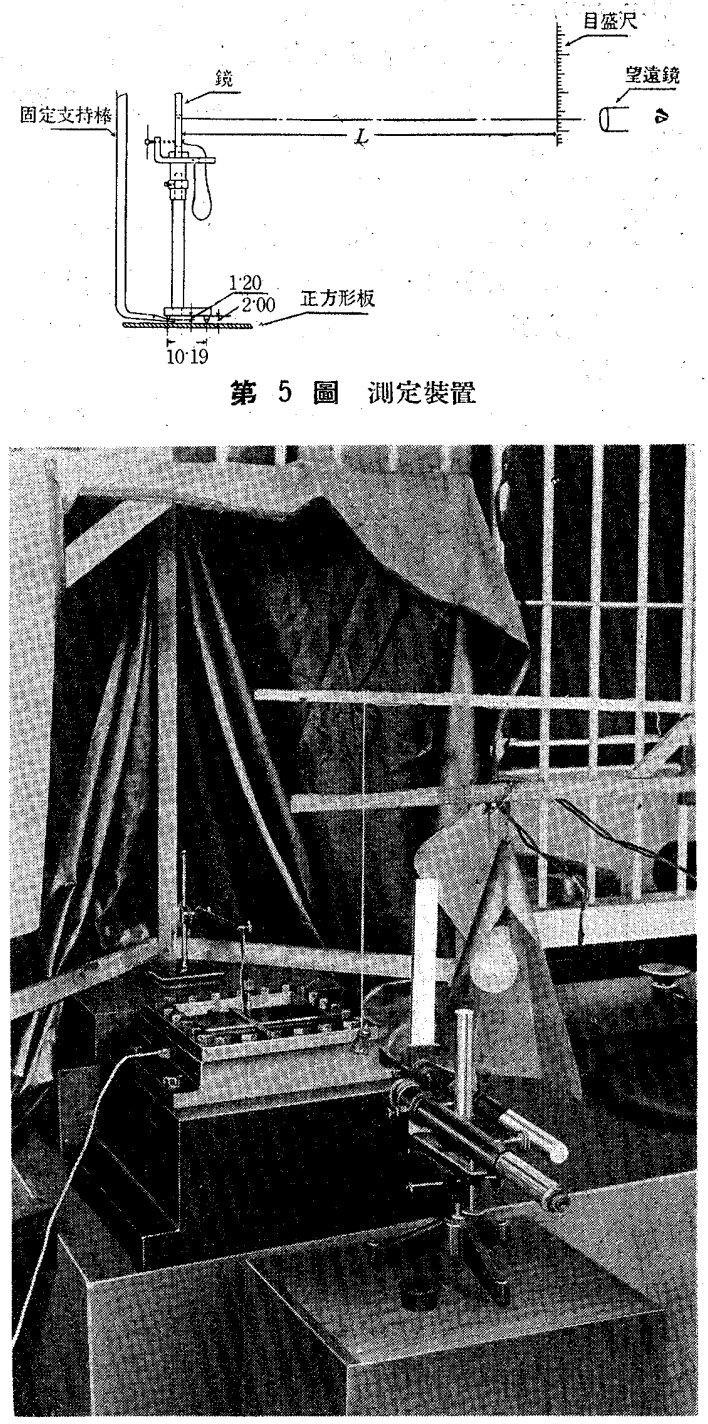

寫 異 $A$

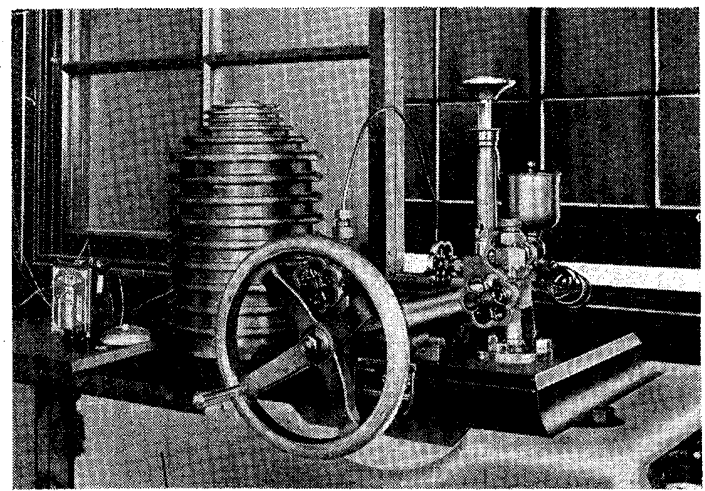

寫 真 $B$

昭 和 7 年 9 月] 
偖本實驗では荷重を $p_{0}=0.5 \mathrm{~kg} / \mathrm{cm}^{2}$ とし、之に相當 寸る撓みを測定した。第 3 表は此實測值を示し、第6 圖 は之を圖示したもので有る。

次に實測せる撓みの曲線を基にして第 7 圖の樣に $\partial w / \partial x, \partial^{2} w / \partial x^{2}$ 及 $\partial w / \partial y, \partial^{2} w / \partial y^{2}$ を求めて、 $y / a=1 / 2$ に於ける彎曲偶力及垂直應力を求を扎ば、第 4, 第 5 表 及第 8 , 第 9 圖の樣になる。少以上の諸表には計算值を 記入して實測值と比較出來る樣にした。

以上の結果から判る樣に計算値と璝测値とは可成良く 一致する。唯今の如き場合では最大應力は板に呼起され るものより梁の方が大きく倍近い值となる。吻論之等兩 者の最大應力が相等しくなる樣にする事が望ましいのは 云ら迄もない。

最後に本實驗を手傅はれた加藤及山本の兩氏に深く感 謝の意を㳖す。

第 3 表 撓州 $w$, 但 $p_{0}=0.5 \mathrm{~kg} / \mathrm{cm}^{2}$ の場合

\begin{tabular}{|c|c|c|c|}
\hline \multirow{2}{*}{$x / a$} & \multirow{2}{*}{$y / a$} & \multicolumn{2}{|c|}{$w, \mathrm{~mm}$} \\
\hline & & 計 算 值 & 项 测 颠 \\
\hline $1 / 8$ & $4 / 8$ & 0.045 & 0.049 \\
\hline $2 / 8$ & $"$ & $0 \cdot 119$ & 0.117 \\
\hline $3 / 8$ & $"$ & $0 \cdot 167$ & 0.169 \\
\hline $4 / 8$ & $"$ & $0 \cdot 188$ & $0 \cdot 185$ \\
\hline $1 / 8$ & $3 / 8$ & 0.041 & 0.043 \\
\hline $2 / 8$ & $"$ & $0 \cdot 108$ & $0 \cdot 111$ \\
\hline $3 / 8$ & $"$ & $0 \cdot 156$ & $0 \cdot 158$ \\
\hline $1 / 8$ & $2 / 8$ & 0.029 & 0.033 \\
\hline $2 / 8$ & $"$ & $\dot{0} \cdot 087$ & 0.085 \\
\hline $1 / 8$ & $1 / 8$ & 0.011 & 0.015 \\
\hline
\end{tabular}
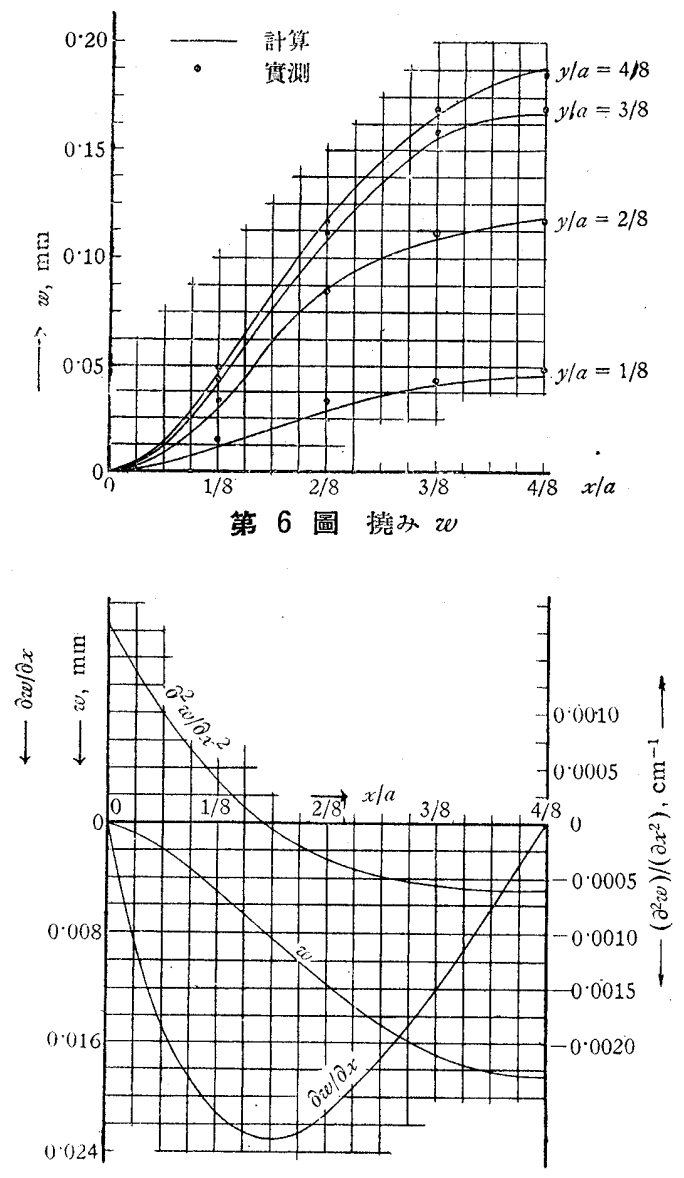

第 7 圖

第 4 表 彎曲偶力 $M_{x}, M_{y}$ 及 $M_{b_{j}}$ ，但 $p_{0}=0.5 \mathrm{~kg} / \mathrm{cm}^{2}$ の場合

\begin{tabular}{|c|c|c|c|c|c|c|c|}
\hline \multirow{2}{*}{$x / a$} & \multirow{2}{*}{$y / a$} & \multicolumn{2}{|c|}{$M_{\infty}, \mathrm{kgcm} / \mathrm{cm}$} & \multicolumn{2}{|c|}{$M_{y}, \mathrm{kgcm} / \mathrm{cm}$} & \multicolumn{2}{|c|}{$M_{b_{0}}, \mathrm{~kg} \mathrm{~cm}$} \\
\hline & & 計算 值 & 貫湘做 & 計算做 & 䫝測值 & 計 算 值 & 赛湘值 \\
\hline 0 & $1 / 2$ & $-8 \cdot 028$ & $-7 \cdot 927$ & $-2 \cdot 407$ & $-2 \cdot 378$ & -23.375 & -23.080 \\
\hline $1 / 8$ & $"$ & -1.648 & - & -0.160 & - & -5.120 & -4.919 \\
\hline $2 / 8$ & $"$ & $+1 \cdot 801$ & - & $+1 \cdot 767$ & -- & +4.066 & +3.973 \\
\hline $3 / 8$ & $"$ & $+3 \cdot 116$ & - & +27834 & - & $+7 \cdot 245$ & +6.950 \\
\hline $4 / 8$ & $"$ & $+3 \cdot 318$ & +3.379 & +3.318 & $+3 \cdot 379$ & $+7 \cdot 430$ & $+7 \cdot 568$ \\
\hline
\end{tabular}

第 5 表 垂望應力 $\sigma_{x}, \sigma_{y}$ 及 $\sigma_{b}$, 但 $p_{0}=0.5 \mathrm{~kg} / \mathrm{cm}^{2}$ の場合

\begin{tabular}{|c|c|c|c|c|c|c|c|}
\hline \multirow{2}{*}{$x / a$} & \multirow{2}{*}{$y / a$} & \multicolumn{2}{|c|}{$\sigma_{x}, \mathrm{~kg} / \mathrm{cn}^{2}$} & \multicolumn{2}{|c|}{$\sigma_{y}, \mathrm{~kg} / \mathrm{cm}^{2}$} & \multicolumn{2}{|c|}{$\sigma_{b_{3},}, \mathrm{~kg} / \mathrm{cm}^{2}$} \\
\hline & & 計算值 & 嘪 測 值 & 計筧值 & 筫 測 值 & 計 算值 & 賽湘 值 \\
\hline 0 & $1 / 2$ & $\mp 607$ & $\mp 599$ & $\mp 182$ & $\mp 180$ & $\left\{\begin{array}{l}-860 \\
+1103\end{array}\right.$ & $\left\{\begin{array}{l}-849 \\
+1088\end{array}\right.$ \\
\hline $1 / 8$ & $"$ & $\mp 125$ & - & $\mp 12 \cdot 1$ & - & $\left\{\begin{array}{l}-189 \\
+\quad 242\end{array}\right.$ & $\left\{\begin{array}{l}-181 \\
+\quad 232\end{array}\right.$ \\
\hline $2 / 8$ & $"$ & \pm 136 & - & \pm 134 & - & $\left\{\begin{array}{r}+150 \\
-192\end{array}\right.$ & $\left\{\begin{array}{l}+146 \\
-187\end{array}\right.$ \\
\hline $3 / 8$ & $"$ & \pm 236 & - & \pm 215 & - & $\left\{\begin{array}{l}+267 \\
-342\end{array}\right.$ & $\left\{\begin{array}{l}+256 \\
-328\end{array}\right.$ \\
\hline $4 / 8$ & $"$ & \pm 251 & \pm 255 & \pm 251 & \pm 255 & $\left\{\begin{array}{l}+274 \\
-351\end{array}\right.$ & $\left\{\begin{array}{r}+278 \\
-357\end{array}\right.$ \\
\hline
\end{tabular}

[第 35 卷第 185 號 


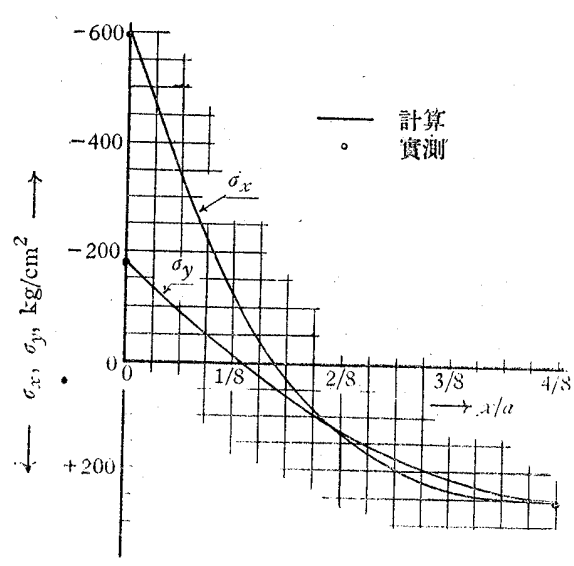

第 8 圖 垂㨁應力 $\sigma_{x}$ 及 $\sigma_{y}$

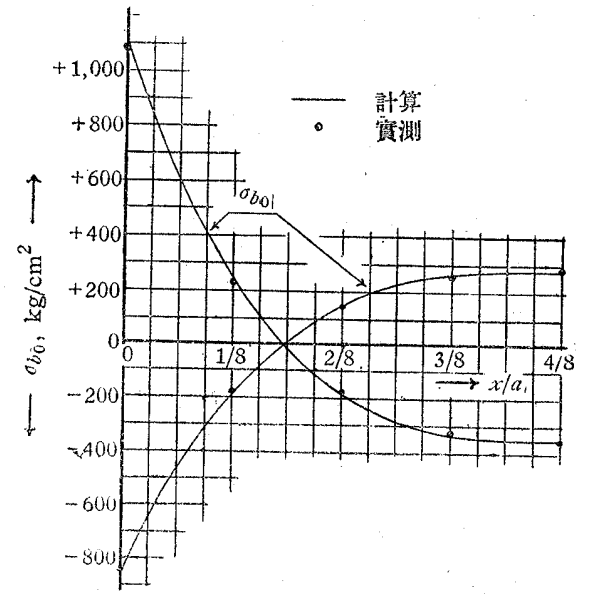

第 9 圖 垂值憵力 $\sigma b_{i j}$

二本板にて压せられたるローラーの

最大剪到内力の分布

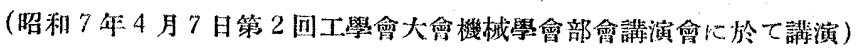

正員王學士 覺前 睦 夫

Die Verteilung der maximalen Shubspannung in einer

Walze, der zwischen zwei parallelen Platten durch die Last zusammengedrückt wird

Von Mutuo KakU\%kN, Kôgakusi, Mitglied

\section{摘要}

本交は H. Hertz の式に估つて、先づローシー內の任意の點に於け当內力を求め、次に其等內力を朋ひて

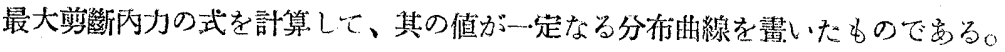

今問題を普通一般に行は扎て居る樣に彈性體の間題と して二次元的に取扱ふ。又ローラーと二平板とは便にて 接觸する篦で女るが、計算の便宜上、線にて接するもの とする。第 1 圖に示されて居る樣に $Y$ を横軸に $Z$ を 縱䡛に取り、 $\sigma_{y}, \sigma_{z}$ を各 $Y$ 軸、 $Z$ 軸の方向に作用する 生縮內力とし、叉 $\tau_{y z}$ を $Y$ 軸に垂㨁なる平面に於て $Z$

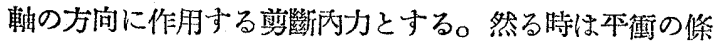
件より $\sigma_{y}, \sigma_{z}$ 及 $\tau_{y z}$ 閒に次の關係式名成立する。

(1) 安治川露工所技師

昭 和 7 年 9 月]

$$
\left.\begin{array}{l}
\partial \sigma_{y} / \partial y+\partial \tau_{y z} / \partial z=0 \\
\partial \sigma_{z} / \partial z+\partial \tau_{y z} / \partial y=0
\end{array}\right\}
$$

$\sigma_{y}, \sigma_{z}$ 攻 $\tau_{y z}$ を內力隶數 $F$ の助によつて表はすと

$$
\left.\begin{array}{l}
\sigma_{y}=\partial^{2} F / \partial z^{2}, \sigma_{z}=\partial^{2} F / \partial y^{2} \\
\tau_{y z}=-\partial^{2} F / \partial y \partial z .
\end{array}\right\}
$$

但し $F$ に對する條件として $F$ は次の關係を滿足しなけ ればなら奴。

$$
\nabla^{4} F=\left(\partial^{2} / \partial y^{2}+\partial^{2} / \partial z^{2}\right)^{2} F=0^{\circ}
$$


new dynamometer devised by the authors, the recording of the cutting force is carried out by a pure mechanical method; a rectangular bar is twisted by the force, and its angular displacement is magnified by means of levers and is recorded. As its construction is very simple it is useful not only in laboratories, but also in workshops. So far as the depression of the tool point is concerned, it is superior to a certain hydraulic dynamometer made in Germany. The said depression in the authors' dynamometer, for example, when the deflection of the recording pen is $20 \mathrm{~mm}$, is $39 \%$ of that in the hydraulic dynamometer at the corresponding deflection of the pen, and 21\% when the oth $\mathrm{r}$ fulculum is used. The capacity of the dynamometer may be easily altered by altering the torsion bar or the ratio of magnification. It is proved by calibration that displacements of the recording pen are in almost perfect rectilinear relation with the loads, and that there is neither hysterisis nor time effect.

In the present paper, the results of measurements are also reported. The experiments were carried out for mild steel, cast iron, gun metal, copper, alminium, bakelite and ebonite at various combinations of feeds and cutting depths, which make the cutting area $1^{*} 26,0^{*} 945,0^{*} 623$ or $0.315 \mathrm{~mm}^{2}$.

(Original p. 914)

\section{THEORY AND EXPERIMENT ON A RECTANGULAR PLATE CLAMPED AT THE PERIPHERY AND SUPPORTED BY MANY ELASTIC BEAMS UNDER UNIFORMLY DISTRIBUTED LOAD} Read before the 2nd General Meeting of the Society of
Engineering, S.M.E. Section, Apr. 7, 1932

By

Chûji FujII, Kôgakusi, Member

Engineer, Yokohama Dock Co. Ltđ., Yokohama

In this paper, an attempt is made to solve the problem of a rectangular plate which is simply supported or perfectly or imperfectly clamped at the periphery and supported by many elastic beams parallel to the edges of plate and subjected to a distributed load in any manner.

To simplify the problem, it may be assumed that the elastic beams lie symmetrically to the. center line of the plate and the latter is subjected to an uniformly distributed load. The reaction, produced by the elastic beams can be considered as the external force acting on the plate, so the differential eqnation of an elastic plane can be written as follows :-

$$
\begin{aligned}
& N \Delta \Delta \omega=p_{0}-E_{a / 2} I_{a / 2} \frac{d^{4} w_{x=a / 2}}{d y^{4}}-E_{b / 2} I_{b / 2} \frac{d^{4} w_{y=b / 2}}{d x^{4}} \\
& -2 \sum_{1,0 . .}^{i}\left[E_{a_{i}} I_{a_{i}} \frac{d^{\prime} w_{x=a_{i}}}{d y^{4}}+E_{b_{i}} I_{b_{i}} \frac{d^{4} w_{y=b_{i}}}{d x^{4}}\right] \\
& \text { here, } \Delta=\frac{\partial^{2}}{\partial x^{2}}+\frac{\partial^{2}}{\partial y^{2}} \\
& \omega=\text { deflection of the plate. } \\
& N=E h^{2} / 12\left(1-\nu^{2}\right) \\
& E=\text { modulus of elasticity } \\
& \nu=\text { Poisson's ratio of the plate. } \\
& h=\text { thickness of the plate, }
\end{aligned}
$$

September, 1932] 
$p_{0}=$ uniform load,

$a, b=$ breath and length of the plate respectively,

$x, y=$ rectangular co-ordinates, the origin being taken at the corner and their directions coincide with sides of the plate,

$E_{a / 2}, E_{a_{i}}, E_{b / 2}, E_{b_{i}}=$ moduli of elasticity of the elastic beams at $x=a / 2, x=a_{i}, y=b / 2$ and $y=b_{i}$ respectively,

$\dot{I}_{a / 2}, I_{a_{i}}, I_{b / 2}, I_{b_{i}}=$ moments of inertia of the elastic beams at $x=a / 2, x=a_{i}, y=b / 2$ and $y=b_{i}$ respectively,

Now the auther assumed that the deflection of the plate can be represented by the following formula,

$$
\begin{gathered}
\omega=\frac{p_{0} a^{4}}{N} \sum_{m}^{\infty} \sum_{n}^{\infty} \frac{A_{m n}}{\left(m^{2}+\lambda^{2} n^{2}\right)^{2}}\left[\frac{1}{m \pi} \cdot \sin \frac{m \pi x}{a}+\alpha\left(\frac{x^{2}}{a^{2}}-\frac{x}{a}\right)\right] \cdot\left[\frac{1}{n \pi} \sin \frac{m \pi y}{b}+\beta\left(\frac{y^{2}}{b^{2}}-\frac{y}{b}\right)\right] . \\
m, n=1,3,5, \cdots
\end{gathered}
$$

In this equation, $\lambda$ is equal to $a / b$ and for $\alpha, \beta$ we put the next limitations, i.e. $0 \leq \alpha \leq 1$, $0 \leq \beta \leq 1$. Therefore, for $\alpha=\beta=0$ or $\alpha=\beta=1$, the equation (2) satisfies the boundary condition of the freely supported or perfectly clamped rectangular plate respectively. But, the equation (2) is not necessarily the solution of eq. (1), hence we can determine the unknown coefficient $A_{m n}$ in eq. (2) as to satisfy this condition.

The auther has introduced the general conditional equation for $A_{m n}$, and for examples, some numerical calculations and experiments on special cases have been carried out. From these sestults, he has confirmed that the theoretieal results well coincide with those of the experiments.

(Original p. 924)

\title{
DIE VERTEILUNG DER MAXIMALEN SCHUBSPANNUNG IN EINER WALZE, DIE ZWISCHEN ZWEI PARALLELEN PLATTEN DURCH DIE LAST ZUSAMMENGEDRUECKT WIRD
}

\author{
Vorgetragen auf der 2. Hauptversammlung des Vereines \\ der Ingenieure, S.M.E. Abteilung, Apr. 7, 1932

$$
\text { Von }
$$ \\ Mutuo Kakuzen, Kôgakusi Mitglied \\ Maschinenbauingenieur, Adikawa Eisen Werke, Ôsaka
}

Es behandelt sich um die Spannungen in irgend einem Punkt in Walze zuerst und nachher die maximale Schubspannung:

Die Spannungen $\sigma_{y}, \sigma_{z}$ und $\tau_{y z}$ lassen sich leicht unter der Benutzung von Spannungsfunktion berechnen und damit wird die in folgender Beziehung stehende maximale Schubspannung $\tau_{\max }$ berechnet,

$$
\begin{aligned}
\tau_{\max }^{2} & =\frac{1}{4}\left(\sigma_{y}-\sigma_{z}\right)^{2}+\tau_{y z}^{2} \\
\text { also, } \tau_{\max }^{2} & =\left(\frac{p}{\pi l}\right)^{2}\left[\frac{\cos ^{2} \varphi_{1}}{r_{1}{ }^{2}}+\frac{\cos ^{2} \varphi_{2}}{r_{2}{ }^{2}}+\frac{2 \cos \varphi_{1} \cos \varphi_{2} \cos 2\left(\varphi_{1}+\varphi_{2}\right)}{r_{1} r_{2}}\right]
\end{aligned}
$$

Die Punkte mit konstantem Wert $K \equiv \tau_{\max } / \frac{p}{\pi l a}$ sind errechnet und mit Kurven zeichnerisch dargestellt. Die Schar von auf dieser Weise erhaltenen K-Kurven stimmt ziemlich gut mit der der schon experimental erhaltenen Isochromen-Kurven in Photoelastizität überein.

(Original p. 931)

[Vol. XXXV, No. 185 